\title{
Topology and arithmetic of resultants, II: The resultant 1 hypersurface
}

\author{
Benson Farb and Jesse Wolfson \\ With an appendix by Christophe Cazanave
}

\begin{abstract}
We consider the moduli space $\mathcal{R}_{n}$ of pairs of monic, degree $n$ polynomials whose resultant equals 1 . We relate the topology of these algebraic varieties to their geometry and arithmetic. In particular, we compute their étale cohomology, the associated eigenvalues of Frobenius, and the cardinality of their set of $\mathbb{F}_{q}$-points. When $q$ and $n$ are coprime, we show that the étale cohomology of $\mathcal{R}_{n / \overline{\mathbb{F}}_{q}}$ is pure, and of Tate type if and only if $q \equiv 1 \bmod n$. We also deduce the values of these invariants for the finite field counterparts of the moduli spaces $\mathcal{M}_{n}$ of $\mathrm{SU}(2)$-monopoles of charge $n$ in $\mathbb{R}^{3}$ and the associated moduli space $X_{n}$ of strongly centered monopoles.

An appendix by Cazanave gives an alternative and elementary computation of the point counts.
\end{abstract}

\section{Introduction}

Consider two monic, degree $n \geqslant 1$, complex polynomials $\phi(z)=z^{n}+a_{n-1} z^{n-1}+\cdots+a_{1} z+a_{0}$ and $\psi(z)=z^{n}+b_{n-1} z^{n-1}+\cdots+b_{1} z+b_{0}$. A beautiful classical fact is that the condition for $\phi$ and $\psi$ to have a common root is polynomial in the coefficients $a_{i}$ and $b_{j}$. More precisely, $\phi$ and $\psi$ have a common root if and only if

$$
\mathcal{R}(\phi, \psi):=\mathcal{R}\left(a_{0}, \ldots, a_{n-1}, b_{0}, \ldots, b_{n-1}\right)=0,
$$

where $\mathcal{R}$ is the resultant, given by

$$
\mathcal{R}(\phi, \psi)=\operatorname{det}\left[\begin{array}{cccccccc}
a_{0} & a_{1} & \cdots & a_{n-1} & 1 & 0 & \cdots & 0 \\
0 & a_{0} & \cdots & \cdots & a_{n-1} & 1 & \cdots & 0 \\
\vdots & \vdots & \vdots & \vdots & \vdots & \vdots & \vdots & \vdots \\
b_{0} & b_{1} & \cdots & b_{n-1} & 1 & 0 & \cdots & 0 \\
0 & b_{0} & \cdots & \cdots & b_{n-1} & 1 & \cdots & 0 \\
\vdots & \vdots & \vdots & \vdots & \vdots & \vdots & \vdots & \vdots \\
0 & 0 & \cdots & b_{0} & \cdots & \cdots & b_{n-1} & 1
\end{array}\right] .
$$

Received 18 August 2015, accepted in final form 9 August 2016.

2010 Mathematics Subject Classification 55R80 (primary), 11G20 (secondary).

Keywords: resultant, monopoles, étale cohomology.

This journal is (C) Foundation Compositio Mathematica 2017. This article is distributed with Open Access under the terms of the Creative Commons Attribution Non-Commercial License, which permits non-commercial reuse, distribution, and reproduction in any medium, provided that the original work is properly cited. For commercial re-use, please contact the Foundation Compositio Mathematica.

B. F. is supported in part by NSF Grant Nos. DMS-1105643 and DMS-1406209. J. W. is supported in part by NSF Grant No. DMS-1400349. 


\section{B. FARB AND J. WOLFSON}

This is a homogeneous polynomial of degree $n$ in the $a_{i}$ and similarly in the $b_{i}$. It has integer coefficients. Fix a field $k$, and denote by $\mathbb{A}^{n}$ the affine space over $k$. The resultant can be thought of as a map

$$
\mathcal{R}: \mathbb{A}^{2 n} \longrightarrow \mathbb{A}^{1}
$$

from the space $\mathbb{A}^{2 n}$ of pairs of monic, degree $n$ polynomials to $k$. The resultant locus $\mathcal{M}_{n}:=$ $\mathbb{A}^{2 n} \backslash \mathcal{R}^{-1}(0)$ is a classically studied object. It is isomorphic to the moduli space of degree $n$ rational maps $\mathbb{P}^{1} \longrightarrow \mathbb{P}^{1}$ taking $\infty$ to 1 . Harder to understand is the "resultant 1 " hypersurface $\mathcal{R}_{n}:=\mathcal{R}^{-1}(1)$ in $\mathbb{A}^{2 n}$.

Since the polynomial $\mathcal{R}$ has integer coefficients, we can extend scalars to $\mathbb{C}$ and consider the complex points $\mathcal{R}_{n}(\mathbb{C})$, and we can also reduce modulo $p$ for any prime $p$. This gives a variety defined over $\mathbb{F}_{p}$, and for any positive power $q=p^{d}$ we can consider both the $\mathbb{F}_{q}$-points and the $\overline{\mathbb{F}}_{q}$-points of $\mathcal{R}_{n}$, where $\overline{\mathbb{F}}_{q}$ is the algebraic closure of $\mathbb{F}_{q}$. Three of the most fundamental arithmetic invariants attached to a such a variety $\mathcal{R}_{n}$ are

(i) the cardinality $\left|\mathcal{R}_{n}\left(\mathbb{F}_{q}\right)\right|$,

(ii) the étale cohomology $H_{\mathrm{et}}^{*}\left(\mathcal{R}_{n / \overline{\mathbb{F}}_{q}} ; \mathbb{Q}_{\ell}\right)$, where $\ell$ is a prime not dividing $q$,

(iii) the eigenvalues of the (geometric) Frobenius morphism

$$
\operatorname{Frob}_{q}: H_{\mathrm{et}}^{*}\left(\mathcal{R}_{n / \overline{\mathbb{F}}_{q}} ; \mathbb{Q}_{\ell}\right) \longrightarrow H_{\mathrm{et}}^{*}\left(\mathcal{R}_{n / \overline{\mathbb{F}}_{q}} ; \mathbb{Q}_{\ell}\right) \text {. }
$$

Our main theorems compute the étale cohomology of $\mathcal{R}_{n}$ as well as the associated eigenvalues of the Frobenius morphism, building on the topological work of Segal and Selby [SS96]. We then apply this to compute the cardinality of finite field versions of these moduli spaces, that is, of $\mathcal{R}_{n}\left(\mathbb{F}_{q}\right)$ and $X_{n}\left(\mathbb{F}_{q}\right)$, where $\mathbb{F}_{q}$ is a finite field.

There is a canonical $\mu_{n}$-action on $\mathcal{R}_{n}$; see the beginning of Section 2 . This induces a $\mu_{n^{-}}$ action on $H_{\mathrm{et}}^{*}\left(\mathcal{R}_{n / \overline{\mathbb{F}}_{q}} ; \mathbb{Q}_{\ell}\right)$. Fix an algebraic closure $\overline{\mathbb{Q}}_{\ell}$. Since $\mathbb{Q}_{\ell}$ has characteristic 0 , it follows that $H_{\text {et }}^{*}\left(\mathcal{R}_{n / \overline{\mathbb{F}}_{q}} ; \overline{\mathbb{Q}}_{\ell}\right):=H_{\text {et }}^{*}\left(\mathcal{R}_{n / \overline{\mathbb{F}}_{q}} ; \mathbb{Q}_{\ell}\right) \otimes_{\mathbb{Q}_{\ell}} \overline{\mathbb{Q}}_{\ell}$ decomposes into a direct sum of irreducible representations of $\mu_{n}$. The irreducible representations of $\mu_{n}$ are parametrized by integers $m$ with $0 \leqslant m<n$, corresponding to $\xi \mapsto \zeta^{m} \xi$, where $\zeta$ is a primitive $n$th root of unity; denote this irreducible representation by $V_{m}$. Let $H_{\text {et }}^{*}\left(\mathcal{R}_{n}\right)_{m}$ denote the isotypic component of $H_{\text {et }}^{*}\left(\mathcal{R}_{n} ; \overline{\mathbb{Q}}_{\ell}\right)$ corresponding to $V_{m}$. Denote by $H_{\mathrm{et}}^{i}\left(\mathcal{R}_{n / \overline{\mathbb{F}}_{q}} ; \mathbb{Q}_{\ell}\right)^{\mu_{n}}$ the subspace of $\mu_{n}$-fixed vectors.

Denote by $\mathbb{Q}_{\ell}(-i)$ the $\operatorname{Gal}\left(\overline{\mathbb{F}}_{q} / \mathbb{F}_{q}\right)$-representation of rank 1 on which the Frobenius morphism acts by $q^{i}$. Also, recall that the étale cohomology of a variety $X$ is pure if the absolute values of the eigenvalues of $\mathrm{Frob}_{q}$ on $H_{\mathrm{et}}^{i}$ are all $q^{i / 2}$; as Deligne showed, this is always the case when $X$ is smooth and projective. The group $H_{\mathrm{et}}^{i}\left(X_{\overline{\mathbb{F}}_{q}} ; \mathbb{Q}_{\ell}\right)$ is of Tate type if the eigenvalues of Frob $_{q}$ are all equal to integer powers of $q$.

Theorem 1.1 (Étale cohomology of $\mathcal{R}_{n}$ ). Let $n \geqslant 1$. For all primes $p$ not dividing $n$, and for all positive powers $q=p^{d}$, the following hold:

(i) The $\ell$-adic cohomology of $\mathcal{R}_{n / \overline{\mathbb{F}}_{q}}$ is pure.

(ii) The $\mu_{n}$-invariants are concentrated in degree 0 :

$$
H_{\mathrm{et}}^{i}\left(\mathcal{R}_{n / \overline{\mathbb{F}}_{q}} ; \mathbb{Q}_{\ell}\right)^{\mu_{n}} \cong \begin{cases}\mathbb{Q}_{\ell}(0), & i=0, \\ 0, & i \neq 0 .\end{cases}
$$

(iii) We have $H_{\mathrm{et}}^{2 i+1}\left(\mathcal{R}_{n / \overline{\mathbb{F}}_{q}} ; \mathbb{Q}_{\ell}\right)=0$ for all $i$. 


\section{The RESUltant 1 HyPersurface}

(iv) For $i>0$, the cohomology $H_{\mathrm{et}}^{2 i}\left(\mathcal{R}_{n / \overline{\mathbb{F}}_{q}} ; \mathbb{Q}_{\ell}\right)$ is nonzero if and only if $i<n$ and $(n-i) \mid n$. In this case, it is of Tate type if and only if $q \equiv 1 \bmod n /(n-i)$. More precisely, let $\mathcal{O}_{a}:=\{1 \leqslant$ $m \leqslant n \mid(m, n)=a\}$. Then we have a Frob $q$-invariant decomposition

$$
H_{\mathrm{et}}^{2 i}\left(\mathcal{R}_{n / \overline{\mathbb{F}}_{q}} ; \overline{\mathbb{Q}}_{\ell}\right) \cong\left(\bigoplus_{m \in \mathcal{O}_{n-i}} \overline{\mathbb{Q}}_{\ell}\right) \otimes_{\mathbb{Q}_{\ell}} \mathbb{Q}_{\ell}(-i),
$$

where Frob $_{q}$ acts on the direct sum by $\left(\overline{\mathbb{Q}}_{\ell}\right)_{m} \stackrel{1}{\longrightarrow}\left(\overline{\mathbb{Q}}_{\ell}\right)_{q m \bmod n}$, where $\left(\overline{\mathbb{Q}}_{\ell}\right)_{m}$ denotes the summand corresponding to a given $m \in \mathcal{O}_{n-i}$. In particular, the following hold:

(a) If $(n-i) \nmid n$, then the rank of $H_{\mathrm{et}}^{2 i}\left(\mathcal{R}_{n / \overline{\mathbb{F}}_{q}} ; \mathbb{Q}_{\ell}\right)$ is 0. If $i<n$ and $(n-i) \mid n$, then $H_{\mathrm{et}}^{2 i}\left(\mathcal{R}_{n / \overline{\mathbb{F}}_{q}} ; \mathbb{Q}_{\ell}\right)$ has rank equal to Euler's totient function $\phi(n /(n-i))$.

(b) The Frob $_{q}$-action is given by the permutation representation coming from the action of Frob $_{q}$ on $\mathcal{O}_{n-i}$.

(c) When $q \not \equiv 1 \bmod n /(n-i)$, the trace of this representation is 0 .

(d) When $q \equiv 1 \bmod n /(n-i)$, the Frob ${ }_{q}$-action on $H_{\mathrm{et}}^{2 i}\left(\mathcal{R}_{n / \overline{\mathbb{F}}_{q}} ; \mathbb{Q}_{\ell}\right)$ is given by multiplication by $q^{i}$, and the trace is $q^{i} \cdot \phi(n /(n-i))$.

Remark 1.2. (i) The variety $\mathcal{R}_{n / \overline{\mathbb{F}}_{q}}$ is smooth (so long as $(q, n)=1$ ) but not projective, and thus purity does not follow from Deligne's proof of the Riemann hypothesis for varieties over finite fields.

(ii) The failure of $H_{\text {et }}^{2 i}\left(\mathcal{R}_{n / \overline{\mathbb{F}}_{q}} ; \mathbb{Q}_{\ell}\right)$ to be of Tate type is precisely the failure of the group scheme $\mu_{n /(n-i)}$ to be of Tate type over $\mathbb{F}_{q}$. See below for details.

Corollary 1.3 (Isotypic decomposition when $q \equiv 1 \bmod n$ ). Let $n \geqslant 1$. Then for $q \equiv 1 \bmod$ $n$, the isotypic decomposition of $H_{\mathrm{et}}^{*}\left(\mathcal{R}_{n / \overline{\mathbb{F}}_{q}} ; \mathbb{Q}_{\ell}\right)$ is Frob $_{q}$-invariant, and for any $1 \leqslant m \leqslant n-1$,

$$
H_{\mathrm{et}}^{2 i}\left(\mathcal{R}_{n / \overline{\mathbb{F}}_{q}}\right)_{m} \cong \begin{cases}\mathbb{Q}_{\ell}(-i), & \operatorname{gcd}(m, n)=n-i, \\ 0, & \text { else. }\end{cases}
$$

Thus, for each $i \geqslant 0$,

$$
H_{\mathrm{et}}^{2 i}\left(\mathcal{R}_{n / \overline{\mathbb{F}}_{q}} ; \mathbb{Q}_{\ell}\right) \cong \bigoplus_{\left\{m \in \mathcal{O}_{n-i}\right\}} \mathbb{Q}_{\ell}(-i)
$$

Note that for $n$ prime and $q \equiv 1 \bmod n$,

$$
H_{\mathrm{et}}^{i}\left(\mathcal{R}_{n / \overline{\mathbb{F}}_{q}} ; \mathbb{Q}_{\ell}\right) \cong \begin{cases}\mathbb{Q}_{\ell}(1-n)^{\oplus(n-1)}, & i=2 n-2, \\ \mathbb{Q}_{\ell}(0), & i=0, \\ 0, & \text { else } .\end{cases}
$$

Theorem 1.1 not only gives the étale cohomology of the varieties $\mathcal{R}_{n}$ but also computes the eigenvalues of the Frobenius morphism acting on these varieties over $\overline{\mathbb{F}}_{q}$. Applying the Grothendieck-Lefschetz trace formula, we conclude the following.

Corollary 1.4 (Cardinality of $\mathcal{R}_{n}\left(\mathbb{F}_{q}\right)$ ). Let $n \geqslant 1$. Then for all primes $p \nmid n$, for each positive power $q$ of $p$, let

Then

$$
\mathcal{F}(q, n):=\left\{a: a \mid n \text { and } q \equiv 1 \bmod \frac{n}{a}\right\}
$$

$$
\left|\mathcal{R}_{n}\left(\mathbb{F}_{q}\right)\right|=q^{2 n-1}\left(\sum_{a \in \mathcal{F}(q, n)} \phi\left(\frac{n}{a}\right) \cdot q^{a-n}\right) .
$$




\section{B. FARB AND J. WOLFSON}

In particular, for $(q-1, n)=1$,

$$
\left|\mathcal{R}_{n}\left(\mathbb{F}_{q}\right)\right|=q^{2 n-1}
$$

and for $q \equiv 1 \bmod n$,

$$
\left|\mathcal{R}_{n}\left(\mathbb{F}_{q}\right)\right|=q^{2 n-1}\left(\sum_{i=0}^{n-1} \phi\left(\frac{n}{n-i}\right) \cdot q^{-i}\right),
$$

where we define Euler's totient function to be identically 0 on $\mathbb{Q} \backslash \mathbb{N}$. When $n$ is prime and $q \equiv 1$ $\bmod n$, this gives

$$
\left|\mathcal{R}_{n}\left(\mathbb{F}_{q}\right)\right|=q^{2 n-1}+(n-1) q^{n} .
$$

We also include an appendix, by Cazanave, in which the above point count (as well as the case when $(q, n) \neq 1)$ is deduced by elementary means, that is, without using étale cohomology.

The results of this paper provide an example of a broader program applying this viewpoint to make concrete calculations for various moduli spaces. For more, see [FW16]. The varieties $\mathcal{R}_{n}$ are closely related to some moduli spaces studied in physics, namely moduli spaces of magnetic monopoles. In Section 3, we apply the results above to deduce similar theorems for these moduli spaces.

Remark on the proofs. One novelty of the proofs in this paper is that we obtain information about various algebraic varieties $Z$ defined over $\mathbb{Z}$ by traversing, in different directions, a "triangle" of viewpoints: arithmetic $\left(\left|Z\left(\mathbb{F}_{q}\right)\right|\right)$, topological $\left(H^{*}(Z(\mathbb{C}) ; \mathbb{Q})\right)$, and geometric $\left(\operatorname{Frob}_{q}\right.$ acting on $\left.H_{\mathrm{et}}^{*}\left(Z_{\overline{\mathbb{F}_{i}}} ; \mathbb{Q}_{\ell}\right)\right)$. As an example, the logic of the starting point of the proofs of Theorem 1.1 and Corollary 1.4 is as follows:

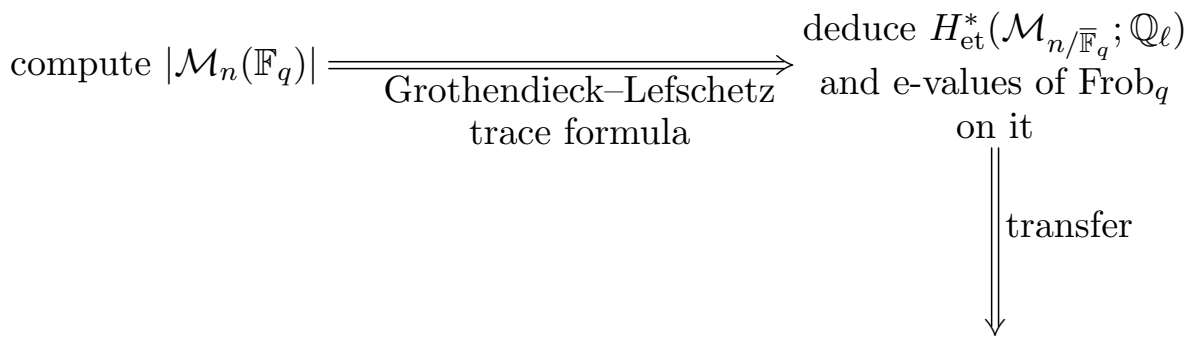

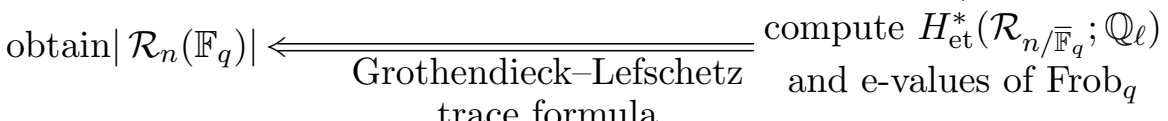

The upper horizontal deduction is a special case of the results of the first paper in this series [FW16]. We take this as a starting point in order to make the remaining deductions.

\section{Proof of Theorem 1.1}

In this section, we prove Theorem 1.1. Throughout this section we fix $n \geqslant 1$ and a prime power $q=p^{d}$ with $d \geqslant 1$.

The $\mu_{n}$-action on $\mathcal{R}_{n}$. The multiplicative group $\mathbb{G}_{m}$ acts on $\mathcal{M}_{n}$ by ${ }^{1}$

$$
\lambda \cdot(\phi, \psi):=(\psi+\lambda(\phi-\psi), \psi) .
$$

\footnotetext{
${ }^{1}$ Note that this action is isomorphic to the one considered by [SS96] under conjugation by the Möbius transformation $z \mapsto z+1$.
} 


\section{The RESUltant 1 HyPersurface}

Because the resultant is homogeneous of degree $n$ with respect to this action, this induces an action of the group $\mu_{n}$ of $n$th roots of unity on $\mathcal{R}_{n}$. We study this action on the étale cohomology of $\mathcal{R}_{n}$.

Recall that the resultant gives a morphism $\mathcal{R}: \mathbb{A}^{2 n} \longrightarrow \mathbb{A}^{1}$. Note that

$$
\mathcal{R}_{1}=\left\{\frac{z+a_{0}}{z+b_{0}}: a_{0}-b_{0}=1\right\} \cong\left\{\frac{z+a_{0}}{z+\left(a_{0}-1\right)}\right\} \cong \mathbb{A}^{1}
$$

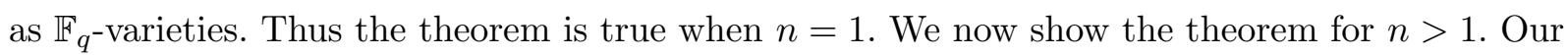
analysis proceeds in a series of steps.

Step 1: the $\mu_{n}$-isotopic decomposition of $H_{\mathrm{et}}^{i}\left(\mathcal{R}_{n / \overline{\mathbb{F}}_{q}} ; \mathbb{Q}_{\ell}\right)$. For each $i \geqslant 0$ there is a decomposition of $H_{\mathrm{et}}^{i}\left(\mathcal{R}_{n / \overline{\mathbb{F}}_{q}} ; \mathbb{Q}_{\ell}\right)$ into $\mu_{n}$-isotypic components:

$$
H_{\mathrm{et}}^{i}\left(\mathcal{R}_{n / \overline{\mathbb{F}}_{q}} ; \mathbb{Q}_{\ell}\right) \cong H_{\mathrm{et}}^{i}\left(\mathcal{R}_{n / \overline{\mathbb{F}}_{q}} ; \mathbb{Q}_{\ell}\right)^{\mu_{n}} \bigoplus H_{\mathrm{et}}^{i}\left(\mathcal{R}_{n / \overline{\mathbb{F}}_{q}}\right)^{\mu_{n}^{\perp}}
$$

where

$$
H_{\mathrm{et}}^{i}\left(\mathcal{R}_{n / \overline{\mathbb{F}}_{q}} ; \overline{\mathbb{Q}}_{\ell}\right)^{\mu_{n}^{\perp}}:=\bigoplus_{m=1}^{n-1} H_{\mathrm{et}}^{i}\left(\mathcal{R}_{n / \overline{\mathbb{F}}_{q}}\right)_{m} .
$$

The decomposition (2.1) is invariant under the action of Frob ${ }_{q}$. However, for $m>0$ the subspace $H_{\text {et }}^{i}\left(\mathcal{R}_{n / \overline{\mathbb{F}}_{q}}\right)_{m}$ is in general not Frob $_{q}$-invariant. In fact, this failure of invariance is at the crux of the proof of the theorem. We begin by finding the Frob $_{q}$-invariant subspaces.

To this end, for any factor $a$ of $n$, define

$$
\mathcal{O}_{a}:=\{m: 1 \leqslant m \leqslant n-1,(m, n)=a\}
$$

and define

$$
H_{\mathrm{et}}^{i}\left(\mathcal{R}_{n / \overline{\mathbb{F}}_{q}}\right)_{a}:=\bigoplus_{m \in \mathcal{O}_{a}} H_{\mathrm{et}}^{i}\left(\mathcal{R}_{n / \overline{\mathbb{F}}_{q}}\right)_{m} .
$$

Fix $n \geqslant 1$ and assume $(q, n)=1$. We claim that for each $i \geqslant 0$ the splitting

$$
H_{\mathrm{et}}^{i}\left(\mathcal{R}_{n / \overline{\mathbb{F}}} ; \overline{\mathbb{Q}}_{\ell}\right)^{\mu_{n}^{\perp}} \cong \bigoplus_{a \mid n} H_{\mathrm{et}}^{i}\left(\mathcal{R}_{n / \overline{\mathbb{F}}_{q}}\right)_{a}
$$

is Frob $_{q}$-equivariant. To see this, note that since $(q, n)=1$, the multiplication by $q$ acts as an automorphism of $\mathbb{Z} / n \mathbb{Z}$ and so preserves the orders of the elements in $\mathbb{Z} / n \mathbb{Z}$. For $m \in \mathcal{O}_{a}$, the order of $m$ in $\mathbb{Z} / n \mathbb{Z}$ equals $n / a$. Thus the order of $q m$ in $\mathbb{Z} / n \mathbb{Z}$ is also $n / a$, and thus the greatest common divisor of $q m \bmod n$ and $n$ equals $a$, proving the claim.

Step 2: the $\mu_{n}$-invariant part of $H_{\text {et }}^{i}\left(\mathcal{R}_{n / \overline{\mathbb{F}}_{q}} ; \mathbb{Q}_{\ell}\right)$. First, note that $\mathcal{R}-1$ is an irreducible polynomial. Indeed, $\mathcal{R}$ is an irreducible polynomial [vdW49, Section 77] and we have the following result.

Lemma 2.1. Let $\Phi\left(x_{1}, \ldots, x_{n}, y_{1}, \ldots, y_{m}\right)$ be an irreducible, bi-homogeneous polynomial of bidegree $(p, q)$. Then $\Phi-1$ is irreducible.

Proof. Suppose $\Phi-1=P Q$. Without loss of generality, we can assume that $P$ and $Q$ are of total degrees $c$ and $d$. Write $P=P_{0}+P_{1}$, where $P_{0}$ is homogeneous of total degree $c$ and $\operatorname{deg}\left(P_{1}\right)<c$, and, similarly, write $Q=Q_{0}+Q_{1}$. Then $c+d=p+q$,

$$
\Phi=P_{0} Q_{0}, \quad \text { and } \quad-1=P_{0} Q_{1}+P_{1} Q_{0}+P_{1} Q_{1} .
$$




\section{B. FARB AND J. WOLFSON}

The irreducibility of $\Phi$ implies that, without loss of generality, we may assume $P_{0}=1$ and thus $P_{1}=0$. Therefore, we have $Q=\Phi-1$ with $Q_{0}=\Phi$ and $Q_{1}=-1$.

It follows that $H_{\text {et }}^{0}\left(\mathcal{R}_{n / \overline{\mathbb{F}}_{q}} ; \mathbb{Q}_{\ell}\right) \cong \mathbb{Q}_{\ell}(0)$. Since this group is generated by the constant function 1, it follows that $H_{\text {et }}^{0}\left(\mathcal{R}_{n / \overline{\mathbb{F}}_{q}} ; \mathbb{Q}_{\ell}\right) \subseteq H_{\text {et }}^{i}\left(\mathcal{R}_{n / \overline{\mathbb{F}}_{q}} ; \mathbb{Q}_{\ell}\right)^{\mu_{n}}$. We now prove the reverse inclusion.

Recall that we have defined $\mathcal{M}_{n}:=\mathbb{A}^{2 n} \backslash \mathcal{R}^{-1}(0)$. The variety $\mathcal{M}_{n}$ admits a free action of the multiplicative group $\mathbb{G}_{m}=\mathrm{GL}_{1}$ via $\lambda \cdot(\phi / \psi):=\lambda \phi / \psi$. Because the group $\mathbb{G}_{m}$ is special (cf. [Gro58, p. 12]), this gives a Zariski-locally trivial fibering

$$
\mathbb{G}_{m} \longrightarrow \mathcal{M}_{n} \longrightarrow \mathcal{M}_{n} / \mathbb{G}_{m} \cong \mathcal{R}_{n} / \mu_{n},
$$

where $\mathcal{M}_{n} / \mathbb{G}_{m}$ is a fiber bundle over $\mathbb{P}^{n-1}$. (The projection $\phi / \psi \mapsto \phi$ induces a $\mathbb{G}_{m}$-equivariant fibering $\mathcal{M}_{n} \longrightarrow\left(\mathbb{A}^{n}-\{0\}\right)$, and $\mathcal{M}_{n} / \mathbb{G}_{m} \longrightarrow \mathbb{P}^{n-1}$ is the quotient of this $\mathbb{G}_{m}$-action.)

Transfer now gives

$$
H_{\mathrm{et}}^{i}\left(\mathcal{R}_{n / \overline{\mathbb{F}}_{q}} ; \mathbb{Q}_{\ell}\right)^{\mu_{n}} \cong H_{\mathrm{et}}^{i}\left(\mathcal{R}_{n} / \mu_{n / \overline{\mathbb{F}}_{q}} ; \mathbb{Q}_{\ell}\right) \cong H_{\mathrm{et}}^{i}\left(\mathcal{M}_{n} / \mathbb{G}_{m / \overline{\mathbb{F}}_{q}} ; \mathbb{Q}_{\ell}\right) .
$$

It is therefore enough to prove that $H_{\text {et }}^{i}\left(\mathcal{M}_{n} / \mathbb{G}_{m / \overline{\mathbb{F}}_{q}} ; \mathbb{Q}_{\ell}\right)$ vanishes except when $i=0$, in which case (as observed above) it is isomorphic to $\mathbb{Q}_{\ell}(0)$. To this end, we apply the Leray spectral sequence in étale cohomology (see, for example, [Mil13, Theorem 12.7]) to the fibering (2.3). This spectral sequence has

$$
E_{2}^{i, j}= \begin{cases}H_{\mathrm{et}}^{i}\left(\mathcal{M}_{n} / \mathbb{G}_{m / \overline{\mathbb{F}}_{q}} ; H_{\mathrm{et}}^{j}\left(\mathbb{G}_{m / \overline{\mathbb{F}}_{q}} ; \mathbb{Q}_{\ell}\right)\right), & i, j \geqslant 0, \\ 0, & \text { else },\end{cases}
$$

and the spectral sequence converges to $H_{\mathrm{et}}^{i}\left(\mathcal{M}_{n / \overline{\mathbb{F}}_{q}} ; \mathbb{Q}_{\ell}\right)$. This cohomology is computed as a special case of [FW16, Theorem 1.2], where in the notation of [FW16], the variety $\mathcal{M}_{n}$ was called Poly ${ }_{1}^{n, 2}$. It is as follows:

$$
H_{\mathrm{et}}^{i}\left(\mathcal{M}_{n / \overline{\mathbb{F}}_{q}} ; \mathbb{Q}_{\ell}\right) \cong \begin{cases}\mathbb{Q}_{\ell}(0), & i=0 \\ \mathbb{Q}_{\ell}(-1), & i=1 \\ 0, & \text { else }\end{cases}
$$

We use this to compute $E_{2}^{i, j}$. To start, we claim that the monodromy action on $H_{\text {et }}^{j}\left(\mathbb{G}_{m / \overline{\mathbb{F}}_{q}} ; \mathbb{Q}_{\ell}\right)$ is trivial. To see this, first recall that

$$
H_{\mathrm{et}}^{j}\left(\mathbb{G}_{m / \overline{\mathbb{F}}_{q}} ; \mathbb{Q}_{\ell}\right) \cong \mathbb{Q}_{\ell}(-j)
$$

for $j=0,1$ and that $H_{\text {et }}^{j}\left(\mathbb{G}_{m / \overline{\mathbb{F}}_{q}} ; \mathbb{Q}_{\ell}\right)$ equals 0 for $j>1$. Over $\mathbb{C}$, we have $\pi_{1}\left(\mathcal{M}_{n} / \mathbb{G}_{m}\right) \cong$ $\pi_{1}\left(\mathcal{R}_{n} / \mu_{n}\right) \cong \mu_{n}$ since $\pi_{1}\left(\mathcal{R}_{n}\right)=0$. Now, use the fact that the $\mu_{n}$-action on $H_{\text {et }}^{j}\left(\mathbb{G}_{m} / \overline{\mathbb{F}}_{q} ; \mathbb{Q}_{\ell}\right)$ is the restriction of the action of $\mathbb{G}_{m}$ induced by left multiplication. Because $H^{0}\left(\mathbb{G}_{m} / \overline{\mathbb{F}}_{q} ; \mathbb{Q}_{\ell}\right)=\mathbb{Q}_{\ell}$, this action is trivial. Therefore, the $\mu_{n}$-action on $H_{\text {et }}^{j}\left(\mathbb{G}_{m / \mathbb{F}_{q}} ; \mathbb{Q}_{\ell}\right)$ is trivial as well. We have thus shown:

$$
E_{2}^{i, j}= \begin{cases}H_{\mathrm{et}}^{i}\left(\mathcal{M}_{n} / \mathbb{G}_{m / \overline{\mathbb{F}}_{q}} ; \mathbb{Q}_{\ell}(-j)\right), & j=0,1, \\ 0, & \text { else } .\end{cases}
$$

The differential $d_{2}^{i, j}: E_{2}^{i, j} \longrightarrow E_{2}^{i+2, j-1}$ thus gives, for each $i \geqslant 0$, a homomorphism

$$
d_{2}^{i, 1}: H_{\mathrm{et}}^{i}\left(\mathcal{M}_{n} / \mathbb{G}_{m / \overline{\mathbb{F}}_{q}} ; \mathbb{Q}_{\ell}(-j)\right) \longrightarrow H_{\mathrm{et}}^{i+2}\left(\mathcal{M}_{n} / \mathbb{G}_{m / \overline{\mathbb{F}}_{q}} ; \mathbb{Q}_{\ell}(1-j)\right) \text {. }
$$




\section{The RESUltant 1 HYPERSURFACE}

Since $E_{2}^{i, j}=0$ for $i>1$ and $j<0$, the only nontrivial differentials occur on the $E_{2}$ page and, for each $i>0$,

$$
H_{\mathrm{et}}^{i}\left(\mathcal{M}_{n / \overline{\mathbb{F}}_{q}} ; \mathbb{Q}_{\ell}\right) \cong \operatorname{ker}\left(d_{2}^{i-1,1}\right) \oplus H_{\mathrm{et}}^{i}\left(\mathcal{M}_{n} / \mathbb{G}_{m / \overline{\mathbb{F}}_{q}} ; \mathbb{Q}_{\ell}(0)\right) / \text { image }\left(d_{2}^{i-1,1}\right),
$$

while $H_{\mathrm{et}}^{0}\left(\mathcal{M}_{n} / \mathbb{G}_{m / \overline{\mathbb{F}}_{q}} ; \mathbb{Q}_{\ell}(0)\right) \cong H_{\mathrm{et}}^{0}\left(\mathcal{M}_{n / \overline{\mathbb{F}}_{q}} ; \mathbb{Q}_{\ell}\right) \cong \mathbb{Q}_{\ell}(0)$.

Equation (2.4) now gives $H_{\text {et }}^{i}\left(\mathcal{M}_{n / \overline{\mathbb{F}}_{q}} ; \mathbb{Q}_{\ell}\right) \cong \mathbb{Q}_{\ell}(-i)$ for $i=0,1$ and that this equals 0 for $i>1$. Now, the target of $d$ on $E_{2}^{i, j}$ is 0 for $i=4 n, 4 n-1$, so these entries vanish. Working backward, starting at $i=4 n$ and working down to $i=1$, we can apply equation (2.5) using that the left-hand side equals 0 to conclude that $H_{\text {et }}^{i}\left(\mathcal{M}_{n} / \mathbb{G}_{m / \mathbb{F}_{q}} ; \mathbb{Q}_{\ell}\right)=0$ for $i \geqslant 1$. This concludes the computation of $H_{\mathrm{et}}^{i}\left(\mathcal{R}_{n / \overline{\mathbb{F}}_{q}} ; \mathbb{Q}_{\ell}\right)^{\mu_{n}}$.

Step 3: the $H_{\mathrm{et}}^{i}\left(\mathcal{R}_{n / \overline{\mathbb{F}}_{q}}\right)_{a}$. In this step, we analyze the individual summands $H_{\mathrm{et}}^{i}\left(\mathcal{R}_{n / \overline{\mathbb{F}}_{q}}\right)_{a}$ of the decomposition in equation (2.2). We will prove that

$$
H_{\mathrm{et}}^{i}\left(\mathcal{R}_{n / \overline{\mathbb{F}}_{q}}\right)_{a} \cong \begin{cases}\left(\bigoplus_{m \in \mathcal{O}_{a}} \overline{\mathbb{Q}}_{\ell}(0)\right) \otimes_{\mathbb{Q}_{\ell}} \mathbb{Q}_{\ell}(a-n), & j-2(n-a)=0, \\ 0, & j \neq 0 .\end{cases}
$$

Given this claim, the bijection

$$
\mathcal{O}_{a} \stackrel{\cong}{\longrightarrow}\left\{m^{\prime} \leqslant \frac{n}{a}: \operatorname{gcd}\left(m^{\prime}, \frac{n}{a}\right)=1\right\}, \quad m \longmapsto \frac{m}{a}
$$

implies that $\left.H_{\text {et }}^{i}\left(\mathcal{R}_{n / \overline{\mathbb{F}}_{q}} ; \mathbb{Q}_{\ell}\right)\right)$ has rank $\phi\left(n /\left(n-\frac{i}{2}\right)\right)$. To prove the claim, recall that for $a \mid n$, we defined

$$
\mathcal{O}_{a}:=\{m: 1 \leqslant m \leqslant n-1,(m, n)=a\} .
$$

For any $m \in \mathcal{O}_{a}$, note that the order of $e^{2 \pi i m / n}$ is $n / a$. For each $a \mid n$, define

$$
Y_{n, a}:=\left\{\frac{\phi}{\psi} \in \mathcal{R}_{n}: \psi(z)=\chi(z)^{n / a} \text { for some } \chi(z) \in k[z], \operatorname{deg}(\chi)=a\right\} .
$$

Over any field $K$ containing a primitive $n$th root of unity, Segal and Selby [SS96, Proposition 2.1] construct an isomorphism ${ }^{2}$

$$
Y_{n, a / K} \cong \mu_{n} \times_{\mu_{a}}\left(\mathcal{R}_{a / K} \times \mathbb{A}_{K}^{n-a}\right) .
$$

In fact, as we now show, these varieties are isomorphic over $K=\mathbb{F}_{q}$ for any $q$.

Proposition 2.2. The isomorphism (2.6) is defined over $\mathbb{F}_{q}$; that is,

$$
Y_{n, a} \cong \mu_{n} \times_{\mu_{a}}\left(\mathcal{R}_{a} \times \mathbb{A}^{n-a}\right)
$$

as $\mathbb{F}_{q \text {-varieties. }}$

Proof. The homogeneity of the resultant implies that $\mathcal{R}\left(\phi, \chi^{n / a}\right)=\mathcal{R}(\phi, \chi)^{n / a}$. Thus, for any $\phi / \psi \in Y_{n, a}$, the resultant $\mathcal{R}(\phi, \chi)$ is an $(n / a)$ th root of unity. Over $\overline{\mathbb{F}}_{q}$, this gives a decomposition

$$
Y_{n, a} \cong \coprod_{\lambda \in \mu_{n / a}} Y_{n, a, \lambda}
$$

${ }^{2}$ While Proposition 2.1 of [SS96] is stated only over the field $\mathbb{C}$, the proof works verbatim over any field $K$ containing a primitive $n$th root of unity. 


\section{B. FARB AND J. WOLFSON}

where

$$
Y_{n, a, \lambda}:=\left\{\frac{\phi}{\psi} \in Y_{n, a}: \psi(z)=\chi(z)^{n / a} \text { with } \mathcal{R}(\phi, \chi)=\lambda\right\} .
$$

Following Segal and Selby, given $\phi / \chi^{n / a} \in Y_{n, a}$, we can write

$$
\phi=\phi_{0} \chi+\phi_{1}
$$

where $\operatorname{deg}\left(\phi_{0}\right)<n-a$ and $\operatorname{deg}\left(\phi_{1}\right)<a$. Using this, the assignment

$$
\frac{\phi}{\chi^{n / a}} \longmapsto\left(\frac{\phi_{1}}{\chi}, \phi_{0}\right)
$$

defines an isomorphism over $\overline{\mathbb{F}}_{q}$,

$$
Y_{n, a, \lambda} \cong \mathcal{R}_{a}^{-1}(\lambda) \times \mathbb{A}^{n-a} .
$$

Now, a primitive $n$th root of unity $\zeta$ gives an isomorphism of $\overline{\mathbb{F}}_{q}$-varieties

$$
\mathcal{R}_{a}^{-1}\left(\zeta^{j a}\right) \stackrel{\zeta^{-j}}{\cong} \mathcal{R}_{a}, \quad \frac{\phi}{\psi} \longmapsto \frac{\zeta^{-j} \phi}{\psi} .
$$

Taken together, these isomorphisms define a $\mu_{n}$-equivariant map

$$
Y_{n, a} \stackrel{\cong}{\longrightarrow} \coprod_{\lambda \in \mu_{n / a}} Y_{n, a, \lambda} \stackrel{\cong}{\longrightarrow} \coprod_{\lambda \in \mu_{n / a}} \mathcal{R}_{a}^{-1}(\lambda) \times \mathbb{A}^{n-a} \stackrel{\sqcup_{j} \zeta^{-j}}{\longrightarrow} \mathcal{R}_{a} \times \mathbb{A}^{n-a},
$$

where the $\mu_{n}$-action on $\mathcal{R}_{a}^{-1}(\lambda)$ factors through the action of $\mu_{a}$. By Galois descent, it suffices to show that the map (2.7) is Frob $_{q}$-equivariant. But, by the definition of the Frobenius morphism, we have

$$
\operatorname{Frob}_{q}\left(\frac{\zeta^{-j} \phi}{\psi}\right)=\zeta^{-j q} \operatorname{Frob}_{q}\left(\frac{\phi}{\psi}\right)
$$

which shows that, for any $\lambda \in \mu_{n / a}$, the square

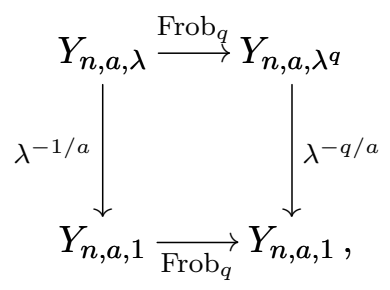

and thus the map (2.7), is Frob $_{q}$-equivariant and thus defined over $\mathbb{F}_{q}$. Combining this with the resultant

$$
\mathcal{R}: Y_{n, a} \longrightarrow \mu_{n / a}
$$

we obtain a map of $\mathbb{F}_{q}$-varieties

$$
Y_{n, a} \stackrel{\pi}{\longrightarrow} \mu_{n / a} \times\left(\mathcal{R}_{a} \times \mathbb{A}^{n-a}\right),
$$

which splits the torsor (2.8) below over $\overline{\mathbb{F}}_{q}$. By inspection, we see that this is an isomorphism over $\overline{\mathbb{F}}_{q}$, and because $\pi$ is Frob $_{q}$-equivariant, $\pi^{-1}$ is as well. Thus we conclude that $\pi$ is an isomorphism over $\mathbb{F}_{q}$.

By inspection, we see that $\pi$ induces an isomorphism of $\mu_{n}$-varieties over $\mathbb{F}_{q}$,

$$
Y_{n, a} \cong \mu_{n} \times_{\mu_{a}}\left(\mathcal{R}_{a} \times \mathbb{A}^{n-a}\right) \text {. }
$$




\section{The RESUltant 1 HyPersurface}

Remark 2.3. We remark that we are using here, in a crucial way, the condition $\mathcal{R}(\phi, \psi)=1$ (as opposed to, say, $\mathcal{R}(\phi, \psi)=2)$, since $\mathcal{R}(\phi, \psi)=\mathcal{R}(\phi, \chi)^{n / a}$, and so $\mathcal{R}(\phi, \chi)$ is an $(n / a)$ th root of unity.

Proposition 2.2 implies that, as $\mu_{n}$-representations,

for each $i \geqslant 0$.

$$
H_{\mathrm{et}}^{i}\left(Y_{n, a / \overline{\mathbb{F}}_{q}} ; \mathbb{Q}_{\ell}\right) \cong \operatorname{Ind}_{\mu_{a}}^{\mu_{n}} H_{\mathrm{et}}^{i}\left(\mathcal{R}_{a / \overline{\mathbb{F}}_{q}} ; \mathbb{Q}_{\ell}\right)
$$

Lemma 2.4. For any $a \mid n$, for every positive power $q$ of $p$, we have

$$
H_{\mathrm{et}}^{*}\left(\left(\mathcal{R}_{n}-Y_{n, a}\right)_{/ \overline{\mathbb{F}}_{q}}\right)_{a}:=\bigoplus_{m \in \mathcal{O}_{a}} H_{\mathrm{et}}^{*}\left(\left(\mathcal{R}_{n}-Y_{n, a}\right)_{/ \overline{\mathbb{F}}_{q}}\right)_{m}=0
$$

Proof. The analogous lemma over $\mathbb{C}$ is [SS96, Proposition 2.2]. We now adapt its proof to the étale setting.

For any $n_{1}, n_{2}>0$ with $n=n_{1}+n_{2}$, let $\mathcal{U}_{n_{1}, n_{2}} \longrightarrow \mathcal{R}_{n}$ be the étale neighborhood defined by

$$
\mathcal{U}_{n_{1}, n_{2}}:=\left\{\left(\phi, \psi_{1}, \psi_{2}\right): \operatorname{deg}\left(\psi_{i}\right)=n_{i} \text { and } \mathcal{R}\left(\psi_{1}, \psi_{2}\right)=\mathcal{R}\left(\phi, \psi_{1} \psi_{2}\right)=1\right\} .
$$

Given $\left(\phi, \psi_{1}, \psi_{2}\right) \in \mathcal{U}_{n_{1}, n_{2}}$, there is a unique decomposition

$$
\frac{\phi}{\psi_{1} \psi_{2}}=\frac{\phi_{1}}{\psi_{1}}+\frac{\phi_{2}}{\psi_{2}}
$$

Let $G$ be the group

$$
G:=\left\{\left(\zeta_{1}, \zeta_{2}\right) \in \mathbb{G}_{m} \times \mathbb{G}_{m}: \zeta_{1}^{n_{1}} \zeta^{n_{2}}=1\right\} .
$$

The group $G$ acts on $\mathcal{U}_{n_{1}, n_{2}}$ via

$$
\left(\zeta_{1}, \zeta_{2}\right) \cdot\left(\phi, \psi_{1}, \psi_{2}\right):=\frac{\zeta_{1} \phi_{1}}{\psi_{1}}+\frac{\zeta_{2} \phi_{2}}{\psi_{2}} .
$$

The action of $\left(\zeta_{1}, \zeta_{2}\right)$ multiplies $\mathcal{R}\left(\phi, \psi_{1} \psi_{2}\right)$ by $\zeta_{1}^{n_{1}} \zeta_{2}^{n_{2}}=1$ since

$$
\begin{aligned}
\mathcal{R}\left(\phi, \psi_{1} \psi_{2}\right) & =\mathcal{R}\left(\phi_{1} \psi_{2}+\phi_{2} \psi_{1}, \psi_{1} \psi_{2}\right) \\
& =(-1)^{n_{1} n_{2}} \mathcal{R}\left(\phi_{1}, \psi_{1}\right) \mathcal{R}\left(\phi_{2}, \psi_{2}\right) \mathcal{R}\left(\psi_{1}, \psi_{2}\right)^{2} .
\end{aligned}
$$

Let $d=\operatorname{gcd}\left(n_{1}, n_{2}\right)$, and let $b$ and $c$ be the integers such that $b n_{1}+c n_{2}=d$. Observe that we have an isomorphism of algebraic groups

$$
G \stackrel{\cong}{\longrightarrow} \mathbb{G}_{m} \times \mu_{d}, \quad\left(\zeta_{1}, \zeta_{2}\right) \longmapsto\left(\zeta_{1}^{c} \zeta_{2}^{-b}, \zeta_{1}^{n_{1} / d} \zeta_{2}^{n_{2} / d}\right) .
$$

The action of $G$ extends the action of $\mu_{n}$, which can be identified with the diagonal subgroup of $G$. Because $H_{\text {et }}^{0}\left(\mathbb{G}_{m} / \overline{\mathbb{F}}_{q} ; \mathbb{Q}_{\ell}\right)=\mathbb{Q}_{\ell}$, for any generator $g \in \mu_{n}$, the power $g^{d}$ acts trivially on $H_{\mathrm{et}}^{*}\left(\mathcal{U}_{n_{1}, n_{2} / \overline{\mathbb{F}}_{q}} ; \mathbb{Q}_{\ell}\right)$; therefore, $H_{\mathrm{et}}^{*}\left(\mathcal{U}_{n_{1}, n_{2} / \overline{\mathbb{F}}_{q}} ; \mathbb{Q}_{\ell}\right)_{m}=0$ for $m \in \mathcal{O}_{a}$ unless $a \mid d$.

To conclude the proof of the lemma, we now observe that $\mathcal{R}_{n}-Y_{n, a}$ admits an étale cover by the collection $\left\{\mathcal{U}_{n_{1}, n_{2}} \mid n=n_{1}+n_{2}\right.$ with $\left.a \nmid n_{1}, n_{2}\right\}$. Using what we just proved as the base case, we now conclude the lemma by induction on the number of étale neighborhoods $\mathcal{U}_{n_{1}, n_{2}}$, using the Mayer-Vietoris sequence in étale cohomology (see, for example, [AM69, Corollary 8.15]).

We apply the long exact sequence of a pair in étale cohomology, see [Mil13, Corollary 16.2], and then we take the direct sum of the $m$-isotypic components for $m \in \mathcal{O}_{a}$. In Milne's notation, setting $c=n-a, Z=Y_{n, a}, X=\mathcal{R}_{n}$, and $U=\mathcal{R}_{n}-Y_{n, a}$, we obtain, for any $0 \leqslant j \leqslant 2(n-a)-2$,

$$
H_{\mathrm{et}}^{j}\left(\mathcal{R}_{n / \overline{\mathbb{F}}_{q}}\right)_{a} \cong H_{\mathrm{et}}^{j}\left(\left(\mathcal{R}_{n}-Y_{n, a}\right)_{/ \overline{\mathbb{F}}_{q}}\right)_{a}
$$




\section{B. FARB AND J. WOLFSON}

and, above degree $2(n-a)-2$, we have a long exact sequence

$$
\begin{aligned}
0 \longrightarrow H_{\mathrm{et}}^{2(n-a)-1}\left(\mathcal{R}_{n / \overline{\mathbb{F}}_{q}}\right)_{a} \longrightarrow H_{\mathrm{et}}^{2(n-a)-1}\left(\left(\mathcal{R}_{n}-Y_{n, a}\right)_{\mathbb{\mathbb { F }}_{q}}\right)_{a} \\
\longrightarrow H_{\mathrm{et}}^{0}\left(Y_{n, a / \overline{\mathbb{F}}_{q}}\right)_{a} \otimes \mathbb{Q}_{\ell}(a-n) \longrightarrow H_{\mathrm{et}}^{2(n-a)}\left(\mathcal{R}_{n / \overline{\mathbb{F}}_{q}}\right)_{a} \\
\longrightarrow H_{\mathrm{et}}^{2(n-a)}\left(\left(\mathcal{R}_{n}-Y_{n, a}\right)_{/ \overline{\mathbb{F}}_{q}}\right)_{a} \cdots H_{\mathrm{et}}^{j-2(n-a)}\left(Y_{n, a / \overline{\mathbb{F}}_{q}}\right)_{a} \otimes \mathbb{Q}_{\ell}(a-n) \\
\longrightarrow H_{\mathrm{et}}^{j}\left(\mathcal{R}_{n / \overline{\mathbb{F}}_{q}}\right)_{a} \longrightarrow H_{\mathrm{et}}^{j}\left(\left(\mathcal{R}_{n}-Y_{n, a}\right)_{/ \overline{\mathbb{F}}_{q}}\right)_{a} \cdots
\end{aligned}
$$

By Lemma 2.4, the terms $H_{\text {et }}^{i}\left(\left(\mathcal{R}_{n}-Y_{n, a}\right)_{/ \overline{\mathbb{F}}_{q}}\right)_{a}$ vanish. It follows that $H_{\text {et }}^{j}\left(\mathcal{R}_{n / \overline{\mathbb{F}}_{q}}\right)_{a}=0$ for $j \leqslant 2(n-a)-1$ and that, for $j \geqslant 2(n-a)$,

$$
H_{\mathrm{et}}^{j}\left(\mathcal{R}_{n / \overline{\mathbb{F}}_{q}}\right)_{a} \cong H_{\mathrm{et}}^{j-2(n-a)}\left(Y_{n, a / \overline{\mathbb{F}}_{q}}\right)_{a} \otimes \mathbb{Q}_{\ell}(a-n) .
$$

We therefore have

$$
\begin{aligned}
& H_{\mathrm{et}}^{j}\left(\mathcal{R}_{n / \overline{\mathbb{F}}_{q}}\right)_{a} \cong H_{\mathrm{et}}^{j-2(n-a)}\left(Y_{n, a / \overline{\mathbb{F}}_{q}}\right)_{a} \otimes \mathbb{Q}_{\ell}(a-n) \\
& \cong \operatorname{Ind}_{\mu_{a}}^{\mu_{n}} H_{\text {et }}^{j-2(n-a)}\left(\mathcal{R}_{a / \overline{\mathbb{F}}_{q}}\right)_{a} \otimes \mathbb{Q}_{\ell}(a-n) \\
& \cong \operatorname{Hom}_{\mu_{n}}\left(\bigoplus_{m \in \mathcal{O}_{a}} V_{m}, \operatorname{Ind}_{\mu_{a}}^{\mu_{n}} H_{\mathrm{et}}^{j-2(n-a)}\left(\mathcal{R}_{a / \overline{\mathbb{F}}_{q}}\right) \otimes \mathbb{Q}_{\ell}(a-n)\right) \\
& \cong \bigoplus_{m \in \mathcal{O}_{a}} \operatorname{Hom}_{\mu_{a}}\left(\operatorname{Res}_{\mu_{a}}^{\mu_{n}} V_{m}, H_{\mathrm{et}}^{j-2(n-a)}\left(\mathcal{R}_{a / \overline{\mathbb{F}}_{q}}\right) \otimes \mathbb{Q}_{\ell}(a-n)\right) \\
& \cong \bigoplus_{m \in \mathcal{O}_{a}} \operatorname{Hom}_{\mu_{a}}\left(V_{0}, H_{\mathrm{et}}^{j-2(n-a)}\left(\mathcal{R}_{a / \overline{\mathbb{F}}_{q}}\right) \otimes \mathbb{Q}_{\ell}(a-n)\right) \\
& \cong \bigoplus_{m \in \mathcal{O}_{a}} H_{\mathrm{et}}^{j-2(n-a)}\left(\mathcal{R}_{a / \overline{\mathbb{F}}_{q}}\right)^{\mu_{a}} \otimes \mathbb{Q}_{\ell}(a-n), \\
& \cong \begin{cases}\left(\bigoplus_{m \in \mathcal{O}_{a}} \overline{\mathbb{Q}}_{\ell}\right) \otimes \mathbb{Q}_{\ell}(a-n), & j-2(n-a)=0, \\
0, & j \neq 0,\end{cases}
\end{aligned}
$$

as shown above, thus proving our claim.

Step 4: the permutation action of $\operatorname{Frob}_{q}$. We complete our analysis of $H_{\mathrm{et}}^{i}\left(\mathcal{R}_{n / \overline{\mathbb{F}}_{q}}\right)_{a}$ as a Frob $_{q}$-module. The analysis of step 3 shows that Frob $_{q}$ acts by $q^{n-a}$ times the action of Frob $_{q}$ on the factor $\bigoplus_{m \in \mathcal{O}_{a}} \overline{\mathbb{Q}}_{\ell}$ of $H_{\mathrm{et}}^{2(n-a)}\left(\mathcal{R}_{n}\right)_{a}$. We claim that this is given by a permutation action

$$
\operatorname{Frob}_{q}:\left(\overline{\mathbb{Q}}_{\ell}\right)_{m} \stackrel{1}{\longrightarrow}\left(\overline{\mathbb{Q}}_{\ell}\right)_{q m} \bmod n,
$$

where $\left(\overline{\mathbb{Q}}_{\ell}\right)_{m}$ denotes the summand corresponding to $m \in \mathcal{O}_{a}$.

Granting this, we conclude that the variety $\mathcal{R}_{n}$ is pure. Further, we see that $\mathcal{R}_{n}$ is of Tate type if and only if if $q \equiv 1 \bmod n$.

To prove the claim, recall that the actions of $\mu_{n}$ and of $\operatorname{Frob}_{q}$ on $H_{\mathrm{et}}^{i}\left(\mathcal{R}_{n / \overline{\mathbb{F}}_{q}}\right)$ do not commute. This is because Frob $_{q}$ acts on $\mu_{n}\left(\overline{\mathbb{F}}_{q}\right)$ as an automorphism. For any vector $v \in H_{\text {et }}^{i}\left(\mathcal{R}_{n / \overline{\mathbb{F}}_{q}}\right)$ and any $\sigma \in \mu_{n}$,

$$
\operatorname{Frob}_{q}(\sigma \cdot v)=\operatorname{Frob}_{q}(\sigma) \cdot \operatorname{Frob}_{q}(v) .
$$

To be more explicit, let $\lambda$ be any primitive $n$th root of 1 in $\overline{\mathbb{F}}_{q}$. Then we can write each element of $\mu_{n}$ as $\lambda^{j}$ for some $0 \leqslant j \leqslant n-1$. The action of $\operatorname{Frob}_{q}$ on $\mu_{n}$ is given by $\operatorname{Frob}_{q}\left(\lambda^{j}\right)=\lambda^{j q}$, 


\section{The RESUltant 1 HyPersurface}

where $j q$ is taken $\bmod n$, and so

$$
\operatorname{Frob}_{q}\left(\lambda^{j} \cdot v\right)=\lambda^{q j} \cdot \operatorname{Frob}_{q}(v)
$$

for any $0 \leqslant j \leqslant n-1$. This, combined with step 3 and Proposition 2.2 above prove the claim.

Step 5: computing the trace of Frob $_{q}$. To conclude the proof, we must compute the trace of $\mathrm{Frob}_{q}$, which by the analysis above equals $q^{n-a}$ times the number of Frob $q$-fixed vectors. The permutation action given in equation $(2.11)$ has a fixed vector precisely when $(q-1) m \equiv 0 \bmod n$, that is, when $(q-1) m / a \equiv 0 \bmod n / a$. If $q \equiv 1 \bmod n / a$, then this equation has no solutions since, by assumption, $(m, n)=a$. It follows that in this case Frob $_{q}$ acts with trace 0 . If $q \equiv 1 \bmod n / a$, then this equation has $\phi(n / a)$ solutions, where $\phi$ is Euler's totient function. In particular, in this case, $\operatorname{Frob}_{q}$ acts on $H_{\mathrm{et}}^{2(n-a)}\left(\mathcal{R}_{n} ; \mathbb{Q}_{\ell}\right)$ by multiplication by $q^{n-a}$.

\section{Moduli space of magnetic monopoles}

The varieties $\mathcal{R}_{n}$ are closely related to some moduli spaces studied in physics, and the results above can be used to deduce arithmetic properties of these spaces, as we now explain.

The moduli space $\mathcal{M}_{n}$ of $\mathrm{SU}(2)$-monopoles of charge $n$ in $\mathbb{R}^{3}$ and the associated moduli space $X_{n}$ of strongly centered monopoles have a rich geometric and topological structure. These complex algebraic varieties have been studied by both physicists and mathematicians; see [SS96] and the references therein.

Let $\mathcal{M}_{n}$ be the moduli space of based $\mathrm{SU}(2)$-monopoles in $\mathbb{R}^{3}$ of charge $n$. Elements of $\mathcal{M}_{n}$ are pairs $(A, \Phi)$, where $A$ is a smooth connection on the trivial $\mathrm{SU}(2)$-bundle $E \longrightarrow \mathbb{R}^{3}$ and $\Phi$ is a smooth section of the vector bundle associated to $E$ via the adjoint representation. The pair $(A, \Phi)$ is a monopole if it satisfies two conditions. First, it must give a solution to the Bogomolnyi equation

$$
* F_{A}=D_{A} \Phi,
$$

where $*$ is the Hodge star operator, $D_{A}$ is the covariant derivative operator defined by $A$, and $F_{A}$ is the curvature of $A$. Second, $(A, \Phi)$ must satisfy a regularity and boundary condition. See, for example, [AH88, Chapter 1] for details. These spaces connect to the present paper because of a different description of $\mathcal{M}_{n}$, due to Donaldson.

As explained, for example, by Manton and Murray, there are many ways of describing $\mathcal{M}_{n}$, each of which leads to the moduli space of degree $n$ rational maps $\mathbb{P}^{1} \longrightarrow \mathbb{P}^{1}$. This is summarized in [MM94] by the following diagram:

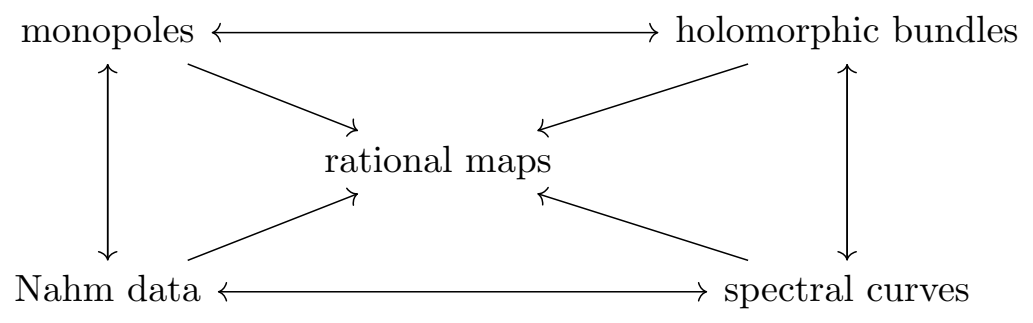

The moduli space $\mathcal{M}_{n}$ is a $2 n$-dimensional complex manifold. Donaldson [Don84] proved that there is a diffeomorphism of $\mathcal{M}_{n}$ with the moduli space of degree $n$ rational maps $\mathbb{P}^{1} \longrightarrow \mathbb{P}^{1}$ that 


\section{B. FARB AND J. WOLFSON}

send $\infty$ to 0 :

$$
\mathcal{M}_{n} \cong\left\{\frac{\phi}{\psi}=\frac{a_{n-1} z^{n-1}+\cdots+a_{1} z+a_{0}}{z^{n}+b_{n-1} z^{n-1}+\cdots+b_{1} z+b_{0}}: \phi, \psi \in \mathbb{C}[z] \text { have no common root }\right\} .
$$

Thus, the diffeomorphism (3.1) endows $\mathcal{M}_{n}$ with the structure of a smooth, complex algebraic variety of (complex) dimension $2 n$. There is a subvariety $X_{n}$ of $\mathcal{M}_{n}$, called the reduced moduli space of SU(2)-monopoles of charge $n$ in $\mathbb{R}^{3}$ or the moduli space of strongly centered monopoles, given by

$$
X_{n} \cong\left\{\frac{\phi}{\psi} \in \mathcal{M}_{n}: b_{n-1}=0 \text { and } \mathcal{R}(\phi, \psi)=1\right\} .
$$

The algebraic variety $X_{n}$ is a smooth hypersurface in $\mathbb{C}^{2 n-1}$. It admits an action of the cyclic group $\mu_{n}$ of $n$th roots unity by algebraic automorphisms. Segal-Selby [SS96] computed the isotypic components of the rational singular cohomology groups $H^{*}\left(X_{n} ; \mathbb{Q}\right)$ under this action.

When $n$ is invertible in $\mathbb{F}_{q}$, there is a Zariski-locally trivial fibration $\mathbb{G}_{a} \longrightarrow \mathcal{R}_{1} \longrightarrow X_{n}$. This together with Theorem 1.4 implies the following.

Corollary 3.1. With notation as above, when $\operatorname{gcd}(q, n)=1$,

$$
H_{\mathrm{et}}^{*}\left(X_{n / \overline{\mathbb{F}}_{q}} ; \mathbb{Q}_{\ell}\right) \cong H_{\mathrm{et}}^{*}\left(\mathcal{R}_{n / \overline{\mathbb{F}}_{q}} ; \mathbb{Q}_{\ell}\right)
$$

as given in Theorem 1.1 and

$$
\left|X_{n}\left(\mathbb{F}_{q}\right)\right|=\left|\mathcal{R}_{n}\left(\mathbb{F}_{q}\right)\right| / q
$$

as given in Corollary 1.4.

\section{ACKNOWLEDGEMENTS}

We are grateful to Sasha Beilinson, Weiyan Chen, Jordan Ellenberg, Matt Emerton, Nir Gadish, Jackson Hance, Sean Howe, Peter May, Joel Specter, Shmuel Weinberger, and Melanie Wood for helpful conversations. We thank Joe Silverman for helpful comments on an earlier draft. We would especially like to thank Christophe Cazanave, not only for contributing the appendix, but also for his many comments, corrections and suggestions; they have greatly improved this paper. Finally, we thank the anonymous referee for many useful comments which greatly improved the paper.

\section{Appendix. An elementary count of the cardinality of $\mathcal{R}_{n}\left(\mathbb{F}_{q}\right)$}

\section{Christophe Cazanave}

We present a more direct approach to count the cardinality of the set $\mathcal{R}_{n}\left(\mathbb{F}_{q}\right)$. It is elementary (no use of étale cohomology is needed), and we obtain a formula valid for all values of $q$ and $n$.

Our main tool is the "addition law" $\oplus$ of pointed rational functions that was introduced in [Caz12, Proposition 3.1]. For the sake of completeness, we recall it briefly.

Let $\mathcal{F}_{n}$ denote the scheme of degree $n$ rational functions which send $\infty$ to $\infty .^{3}$ Given two degrees $n_{1}, n_{2} \geqslant 0$, we define a map

$$
\oplus: \mathcal{F}_{n_{1}} \times \mathcal{F}_{n_{2}} \longrightarrow \mathcal{F}_{n_{1}+n_{2}}
$$

\footnotetext{
${ }^{3}$ Note that $\mathcal{F}_{n}$ is isomorphic to $\mathcal{M}_{n}$ under the Möbius transformation $A(z) / B(z) \mapsto(B(z)-A(z)) / B(z)$.
} 


\section{The RESUltant 1 HyPersurface}

as follows. Two rational functions $A_{i} / B_{i} \in \mathcal{F}_{n_{i}}$, for $i=1,2$, uniquely define two pairs $\left(U_{i}, V_{i}\right)$ of polynomials with $\operatorname{deg} U_{i} \leqslant n_{i}-2$ and $\operatorname{deg} V_{i} \leqslant n_{i}-1$ and satisfying Bézout identities $A_{i} U_{i}+$ $B_{i} V_{i}=1$ (this is true over any ring because $A_{i}$ is monic). Define polynomials $A_{3}, B_{3}, U_{3}$, and $V_{3}$ as

$$
\left[\begin{array}{cc}
A_{3} & -V_{3} \\
B_{3} & U_{3}
\end{array}\right]:=\left[\begin{array}{cc}
A_{1} & -V_{1} \\
B_{1} & U_{1}
\end{array}\right] \cdot\left[\begin{array}{cc}
A_{2} & -V_{2} \\
B_{2} & U_{2}
\end{array}\right]
$$

One easily checks that $A_{3} / B_{3}$ is in $\mathcal{F}_{n_{1}+n_{2}}$. Over a field, this addition law is closely related to the continued fraction expansion of rational functions. This gives the following result.

Lemma A.1. Let $k$ be any field, and let $A / B$ be an element of $\mathcal{F}_{n}(k)$. Then there exist a unique family of monic polynomials $P_{1}, \ldots, P_{r}$ and a unique family of scalars $a_{1}, \ldots, a_{r} \in k^{\times}$such that

$$
\frac{A}{B}=\frac{P_{1}}{a_{1}} \oplus \cdots \oplus \frac{P_{r}}{a_{r}} .
$$

Moreover, the following hold:

(i) Let $n_{i}$ denote the degree of $P_{i}$ for $i \leqslant i \leqslant r$. Then $n=n_{1}+\cdots+n_{r}$.

(ii) There exists a sign $\varepsilon(\underline{n})= \pm 1$ (depending only on the $n_{i}$ ) such that

$$
\mathcal{R}(A, B)=\varepsilon(\underline{n}) a_{1}^{n_{1}} \cdots a_{r}^{n_{r}} .
$$

Proof. The decomposition of $A / B$ as a $\oplus$-sum of polynomials is explained in [Caz12, Example 3.3]. The formula expressing the resultant $\mathcal{R}(A, B)$ in terms of the resultants $\mathcal{R}\left(P_{i}, a_{i}\right)$ can be seen by induction by noting that if $P$ is a monic polynomial of degree $d$ and $a \in k^{\times}$and $A / B \in \mathcal{F}_{n}(k)$, one has

$$
\mathcal{R}\left(\frac{P}{a} \oplus \frac{A}{B}\right)=(-1)^{n d} a^{d} \mathcal{R}(A, B) .
$$

Remark A.2. The precise expression of the sign $\varepsilon$ will not be needed further on. It will be enough to note that when all the $n_{i}$ are even, the $\operatorname{sign} \varepsilon(\underline{n})$ is equal to 1 .

Over a field $k$, specifying a pointed degree $n$ rational function $f=A / B$ such that $\mathcal{R}(A, B)=1$ is thus equivalent to specifying

- an integer $1 \leqslant r \leqslant n$,

- an ordered decomposition $n=n_{1}+\cdots+n_{r}$ (with integers $n_{i} \geqslant 1$ ),

- monic polynomials $P_{1}, \ldots, P_{r}$ of degrees $n_{1}, \ldots, n_{r}$, respectively, and

- units $a_{1}, \ldots, a_{r} \in k^{\times}$such that $a_{1}^{n_{1}} \cdots a_{r}^{n_{r}} \varepsilon(\underline{n})=1$.

We now specialize to $k=\mathbb{F}_{q}$. For $x \in \mathbb{F}_{q}^{\times}$, let us denote by $\mathcal{C}(\underline{n}, x)$ the cardinality of the finite set $\left\{\left(a_{1}, \ldots, a_{r}\right) \in\left(\mathbb{F}_{q}^{\times}\right)^{r}: a_{1}^{n_{1}} \cdots a_{r}^{n_{r}}=x\right\}$. We therefore have

$$
\left|\mathcal{R}_{n}\left(\mathbb{F}_{q}\right)\right|=\sum_{r=1}^{n} \sum_{n=n_{1}+\cdots+n_{r}} q^{n} \mathcal{C}(\underline{n}, \varepsilon(\underline{n})) .
$$

(The factor $q^{n}=q^{n_{1}+\cdots+n_{r}}$ counts the choices for the polynomials $P_{i}$.)

The following lemma is useful.

Lemma A.3. Let $\underline{n}=\left\{n_{1}, \ldots, n_{r}\right\}$ as above. Then

(i) one has $\mathcal{C}(\underline{n}, 1)=(q-1)^{r-1} \operatorname{gcd}\left(q-1, n_{1}, \ldots, n_{r}\right)$;

(ii) if one of the $n_{i}$ is odd, one has the equality $\mathcal{C}(\underline{n}, 1)=\mathcal{C}(\underline{n},-1)$. 


\section{B. FARB AND J. WOLFSON}

Proof. (i) Recall that the group of units $\mathbf{F}_{q}^{\times}$is cyclic of order $q-1$. Therefore, $\mathcal{C}(\underline{n}, 1)$ is also the number of solutions $\left(x_{1}, \ldots, x_{r}\right) \in(\mathbb{Z} /(q-1) \mathbb{Z})^{r}$ of the linear equation

$$
n_{1} x_{1}+\cdots+n_{r} x_{r}=0 .
$$

To count the number solutions of this equation, one can use the invertible changes of variables dictated by the Euclidean algorithm to reduce it to the equivalent equation $\operatorname{gcd}\left(n_{1}, \ldots, n_{r}\right) x_{1}=0$.

(ii) Suppose that $n_{i}$ is an odd integer. Then one has an explicit bijection between the two sets in question given by $a_{i} \mapsto-a_{i}$.

Combining Remark A.2 and Lemma A.3, we get rid of the signs $\varepsilon(\underline{n})$ to obtain

$$
\left|\mathcal{R}_{n}\left(\mathbb{F}_{q}\right)\right|=q^{n} \sum_{r=1}^{n} \sum_{n=n_{1}+\cdots+n_{r}}(q-1)^{r-1} \operatorname{gcd}(q-1, \underline{n}) .
$$

For a fixed $r$, all decompositions $\underline{n}$ with same gcd contribute equally, so we regroup these as follows.

Notation A.4. Let $n, r \geqslant 1$ be two integers.

- Let $\pi_{r}(n)$ be the number of decompositions of $n$ of length $r$. One has $\pi_{r}(n)=\left(\begin{array}{c}n-1 \\ r-1\end{array}\right)$.

- For $d$ a divisor of $n$, let $\pi_{r}(n, d)$ be the number of length $r$ decompositions of $n$ with gcd equal to $d$. One has $\pi_{r}(n, d)=\pi_{r}(n / d, 1)$.

From the identity $\pi_{r}(n)=\sum_{d \mid n} \pi_{r}(n, d)=\sum_{d \mid n} \pi_{r}(n / d, 1)$, the Möbius inversion formula gives $\pi_{r}(\cdot, 1)=\mu \star\left(\begin{array}{c}\cdot-1 \\ r-1\end{array}\right)$, where $\mu$ is the Möbius function and $\star$ denotes the Dirichlet convolution product. Inserting this into equation (A.2) leads to

$$
\left|\mathcal{R}_{n}\left(\mathbb{F}_{q}\right)\right|=q^{n}\left[\mu \star\left(\sum_{r=1}^{n} q^{r-1}\left(\begin{array}{c}
\cdot-1 \\
r-1
\end{array}\right)\right) \star \operatorname{gcd}(\cdot, q-1)\right](n)=q^{n}\left[\mu \star q^{\cdot-1} \star \operatorname{gcd}(\cdot, q-1)\right](n) .
$$

We have thus proved the following theorem.

Theorem A.5. Let $n$ be any positive integer, and let $q$ be any prime power. Then

$$
\left|\mathcal{R}_{n}\left(\mathbb{F}_{q}\right)\right|=q^{n}\left(\sum_{a b c=n} \mu(a) q^{b-1} \operatorname{gcd}(c, q-1)\right) .
$$

We conclude by briefly showing that the point count of Theorem A.5 coincides with that of Corollary 1.4. The two formulas are similar. The only point is to check the following lemma.

Lemma A.6. Let $m \geqslant 1$ be an integer, and let $\delta_{m}: \mathbb{N} \longrightarrow \mathbb{N}$ be the function

$$
\delta_{m}: n \mapsto \begin{cases}1 & \text { if } n \mid m \\ 0 & \text { otherwise }\end{cases}
$$

Then whe have the identity $\delta_{m} \phi=\mu \star \operatorname{gcd}(\cdot, m)$.

Proof. Let $n \geqslant 1$ be an integer. We prove that these two functions agree on $n$. We distinguish cases according to whether $n$ divides $m$ or not.

In the first case, the function $\operatorname{gcd}(\cdot, m)$ coincides with the identity and the equality follows from $\phi=\mu \star \mathrm{id}$. 


\section{The RESUltant 1 HYPERSURFACE}

In the second case, we have to show that

$$
\sum_{d \mid n} \mu(d) \operatorname{gcd}(d, m)=0 .
$$

Replacing $m$ by $\operatorname{gcd}(n, m)$ does not change the value, so one can assume that $m$ divides (strictly) $n$. Let $p$ be a prime dividing $n / m$. We conclude, noting that

- in the above sum, only the divisors without square factor have a nonzero contribution;

- the remaining divisors can be split into those that are divisible by $p$ and those that are not. These two sets have opposite contributions in the sum.

Remark A.7. Given $x \in \mathbb{F}_{q}^{\times}$, we could in the same way write a formula for the number of pointed degree $n$ rational functions $A / B \in \mathcal{F}_{n}\left(\mathbb{F}_{q}\right)$ with resultant $\mathcal{R}(A, B)=x$. This number equals

$$
q^{n}\left(\sum_{a b c=n} \mu(a) q^{b-1} g(c)\right)
$$

where $o$ is the order of $x$ in $\mathbb{F}_{q}^{\times}$and $g: \mathbb{N} \longrightarrow \mathbb{N}$ is the function

$$
g: n \mapsto \begin{cases}\operatorname{gcd}(n, q-1) & \text { if } \operatorname{gcd}(n, q-1) \text { divides } \frac{q-1}{o}, \\ 0 & \text { otherwise }\end{cases}
$$

However, it is interesting to note that, as a function of $x$, this cardinal always reaches its maximum at $x=1$. This is because the functions $\mathcal{C}(\underline{n}, r)$ also reach their maximum at $x=1$. The reason comes from linear algebra: a homogeneous linear equation $a_{1} x_{1}+\cdots+a_{r} x_{r}=0$ has always at least as many solutions as the corresponding inhomogeneous one $a_{1} x_{1}+\cdots+a_{r} x_{r}=k$.

\section{REFERENCES}

AH88 M. Atiyah and N. Hitchen, The geometry and dynamics of magnetic monopoles, M. B. Porter Lectures (Princeton Univ. Press, Princeton, NJ, 1988); https://doi .org/10.1515/9781400859306.

AM69 M. Artin and B. Mazur, Etale homotopy, Lecture Notes in Math., vol. 100 (Springer-Verlag, Berlin, 1969); https://doi.org/10.1007/BFb0080957.

Caz12 C. Cazanave, Algebraic homotopy classes of rational functions, Ann. Sci. Éc. Norm. Supér. (4) 45 (2012), no. 4, 511-534.

Don84 S. K. Donaldson, Nahm's equations and the classification of monopoles, Comm. Math. Phys. 96 (1984), no. 3, 387-407; https://doi.org/10.1007/BF01214583.

FW16 B. Farb and J. Wolfson, Topology and arithmetic of resultants, I, New York J. Math. 22 (2016), 801-821; http://nyjm.albany.edu/j/2016/22-37.html.

Gro58 A. Grothendieck, Torsion homologique et sections rationnelles, Séminaire Claude Chevalley 3 (1958), no. 5, 1-29; http://www. numdam.org/item?id=SCC_1958__3_A5_0.

Mil13 J. Milne, Lectures on étale cohomology, 2013, version 2.21, available at http://www.jmilne.org/ math/CourseNotes/LEC.pdf.

MM94 N. S. Manton and M. K. Murray, Symmetric monopoles, 1994, arXiv:hep-th/9407102.

SS96 G. Segal and A. Selby, The cohomology of the space of magnetic monopoles, Comm. Math. Phys. 177 (1996), no. 3, 775-787; https://doi.org/10.1007/BF02099547.

vdW49 B. L. van der Waerden, Modern algebra (Ungar, New York, 1949). 


\section{B. FARB AND J. WOLFSON}

Benson Farb farb@math.uchicago.edu

Department of Mathematics, University of Chicago, USA

Jesse Wolfson wolfson@math.uchicago.edu

Department of Mathematics, University of Chicago, USA

Christophe Cazanave cazanave@unice.fr

Université Côte d'Azur, CNRS, Laboratoir J. A. Dieudonné, France 